\title{
Autoavaliação de saúde e fatores associados em adolescentes do Estado de Santa Catarina, Brasil
}

\author{
Self-rated health and associated factors in adolescents from Santa Catarina, Brazil
}

Thiago Ferreira de Sousa ${ }^{1}$, Kelly Samara da Silva', Leandro Martin T. Garcia', Giovâni Firpo Del Duca ${ }^{1}$, Elusa Santina A. de Oliveira ${ }^{1}$, Markus Vinicius Nahas ${ }^{2}$

\section{RESUMO}

Objetivo: Identificar a prevalência e os fatores associados à autopercepção negativa de saúde em adolescentes catarinenses.

Métodos: Foi realizado um estudo de delineamento transversal com adolescentes do ensino médio das escolas públicas do Estado de Santa Catarina $(n=5.028)$. A autopercepção negativa de saúde foi analisada em função das variáveis: sexo, renda familiar, prática de atividade física, tabagismo, etilismo, drogas ilícitas e autopercepção de estresse. Aplicouse a regressão logística binária para medidas de associação.

Resultados: A prevalência de autopercepção negativa de saúde foi maior nas moças $(17,6 \%)$ em relação aos rapazes (9,6\%; $p<0,0001)$. Valores superiores de Odds Ratio (OR) foram observados para as moças (OR 1,99; IC95\% 1,66$2,38)$, adolescentes pertencentes às famílias de menor renda (OR 1,54; IC95\% 1,08-2,20), fumantes (OR 2,00; IC95\% $1,35-2,94)$ e que referiram autopercepção negativa de estresse (OR 3,36; IC95\% 2,74-4,12).

Conclusões: Os adolescentes do sexo feminino, os de menor renda familiar, além dos fumantes e os que perceberam negativamente o estresse apresentaram níveis de autopercepção negativa de saúde superiores aos dos seus pares.

Palavras-chave: nível de saúde; conduta de saúde; adolescente.

Instituição: Núcleo de Pesquisa em Atividade Física e Saúde do Centro de Desportos da Universidade Federal de Santa Catarina (UFSC), Florianópolis, SC, Brasil

'Pós-graduando do Programa de Pós-Graduação em Educação Física da Universidade Federal de Santa Catarina (UFSC), Florianópolis, SC, Brasil ${ }^{2}$ Doutor em Educação Física pela University of Southern California, Estados Unidos; Professor do Programa de Pós-Graduação em Educação Física da UFSC, Florianópolis, SC, Brasil

\section{ABSTRACT}

Objective: To identify the prevalence and factors associated with negative self-rated health among adolescents in the State of Santa Catarina, Brazil.

Methods: A cross-sectional investigation was conducted with a representative sample of 5,028 adolescents who were attending public highschools. The negative self-rated health was analyzed in relation to the following variables: gender, income, physical activity practice, smoking, alcohol consumption, drug use and self-rated stress. The binary logistic regression was carried out to test associations.

Results: The prevalence of negative self-rated health was higher among girls (17.6\%) in comparison to boys (9.6\%; $p<0.0001)$. Higher values of $O d d s$ Ratio (OR) were associated with female gender (OR 1.99; 95\% CI 1.66-2.38), lower socioeconomic status (OR 1.54; 95\% CI 1.08-2.20), smoking (OR 2.00; 95\% CI 1.35-2.94), and negative self-rated stress (OR 3.36; 95\%CI 2.74-4.12).

Conclusions: Female adolescents, those with lower socioeconomic status, smokers and those who negatively perceived stress presented higher levels of negative self-rated health.

Key-words: health status; health behavior; adolescent. Introdução

Endereço para correspondência:

Thiago Ferreira de Sousa

Campus Universitário - Trindade - UFSC

CEP 88040-900 - Florianópolis/SC

E-mail: tfsousa_thiago@yahoo.com.br

Fonte financiadora: Conselho Nacional de Desenvolvimento Científico e Tecnológico (CNPq), processo n. ${ }^{\circ}$ 462799/00-0

Conflitos de interesse: nada a declarar

Recebido em: 10/9/2009

Aprovado em: 26/1/2010 


\section{Introdução}

A autopercepção de saúde representa uma importante medida de avaliação do estado de saúde e tem sido bastante utilizada em estudos de base populacional ${ }^{(1,2)}$. Apesar da subjetividade, pesquisadores encontram associação com diferentes comportamentos indesejáveis à saúde ${ }^{(1)}$, com morbidades $^{(3)}$ e predição de mortalidade ${ }^{(4,5)}$.

Na população brasileira adulta e idosa, informações sobre a autopercepção de saúde foram coletadas por meio do sistema de vigilância de fatores de risco e proteção nacional ${ }^{(2)}$. Entretanto, em adolescentes brasileiros, essas informações são escassas e limitadas ${ }^{(6)}$, existindo algumas observações sobre o nível de percepção de saúde em estudos internacionais ${ }^{(7,8)}$.

Os estudos que investigaram a autoavaliação de saúde em adolescentes demonstraram resultados semelhantes às informações obtidas com a população adulta, revelando maior autopercepção de saúde negativa entre os adolescentes pertencentes a famílias de menor renda ${ }^{(7)}$, com elevado consumo de bebidas alcoólicas $^{(9)}$, tabagismo ${ }^{(8)}$, uso de drogas ilícitas ${ }^{(10)}$, menor prática de atividade física ${ }^{(11)} \mathrm{e}$ autopercepção negativa de estresse ${ }^{(12)}$.

A relevância deste estudo está na possibilidade de preencher lacunas de conhecimento no contexto nacional, sendo um indicador que poderá auxiliar em estratégias de ações em Saúde Pública na adolescência. Nesse sentido, o objetivo do presente trabalho foi identificar a prevalência e os fatores associados à autopercepção negativa de saúde em adolescentes catarinenses.

\section{Métodos}

O presente estudo é parte do levantamento Estilo de Vida e Comportamentos de Saúde dos Jovens Catarinenses, realizado em 2001 e 2002 pelo Núcleo de Pesquisa em Atividade Física e Saúde da Universidade Federal de Santa Catarina (UFSC). O delineamento da pesquisa foi transversal, envolvendo adolescentes do ensino médio, de 15 a 19 anos, matriculados nas escolas públicas do Estado de Santa Catarina. No Estado, existiam 26 Unidades Regionais de Educação, totalizando 205.543 jovens matriculados no ensino médio, conforme informações da Secretaria da Educação ${ }^{(13)}$. A amostra foi estratificada pelas Unidades Regionais Oeste, Planalto Serrano, Norte, Vale do Itajaí, Litoral e Sul, com sorteios em dois estágios: o primeiro considerou o porte das escolas (escolas grandes, com 500 alunos ou mais; médias, com 200 a 499 alunos; e pequenas, com, no máximo, 200 alunos) e o segundo, o número de turmas, considerando a proporcionalidade dos alunos conforme o turno de estudo (manhã, tarde ou noite).
O cálculo amostral foi realizado considerando-se as prevalências desconhecidas dos fenômenos a serem investigados $(p=50 \%)$, intervalo de confiança de 95\% (IC95\%) e erro amostral de dois pontos percentuais, obtendo-se um número igual a 2.400 escolares. Tendo em vista a realização da coleta pelo procedimento de conglomerados, utilizou-se o efeito de delineamento igual a dois (efeito do desenho amostral, deff) e foram acrescidos $25 \%$ para os possíveis casos de perdas ou recusas durante a coleta, totalizando uma amostra final igual a 6.000 escolares.

A equipe de coleta foi composta por professores e acadêmicos de Educação Física previamente treinados e sob a supervisão dos pesquisadores responsáveis pelo projeto. Os escolares das turmas sorteadas que estavam na sala de aula no dia da coleta foram informados sobre os objetivos da pesquisa e o anonimato das informações. Após consentimento de participação, o questionário foi aplicado em sala de aula (média de 25 alunos), com duração de 30 a 40 minutos.

$\mathrm{Na}$ coleta de dados, foi utilizado o questionário Compac (Comportamento do Adolescente Catarinense), construído a partir de outros instrumentos de uso internacional. A variável dependente deste estudo foi a autopercepção de saúde, em que os adolescentes respondiam a questão: "Em geral, você considera sua saúde: (excelente, boa, regular ou ruim)”. Posteriormente, consideraram-se as afirmações "excelente/ boa" como autopercepção de saúde positiva e "regular/ruim" como autopercepção de saúde negativa.

As variáveis independentes investigadas foram as demográficas e econômicas: sexo (masculino ou feminino), faixa etária (15 e 16 anos; 17 a 19 anos) e renda familiar mensal ( $\leq \mathrm{R} \$$ 500,$00 ; \mathrm{R} \$ 501,00$ a $1.000,00 ; \mathrm{R} \$ 1.001,00$ a $2.000,00$; $\geq \mathrm{R} \$ 2.000,00$ ); variáveis comportamentais: prática de atividade física moderada a vigorosa (ativos: $\geq 300$ minutos/ semana; insuficientemente ativos: $<300$ minutos/semana, conforme Prochaska et al. ${ }^{14}$ ); tabagismo (fumante, ex-fumante e não-fumante) e consumo de bebidas alcoólicas (sim e não), independentemente da quantidade e frequência; uso de drogas ilícitas alguma vez na vida (não, sim, não quis responder) e percepção subjetiva de estresse (positiva: raramente/às vezes; negativa: quase sempre/excessivamente estressado(a)).

Essa análise secundária dos dados foi conduzida no programa estatístico Statistical Package for the Social Sciences (SPSS) para Windows, versão 16.0. Além da análise descritiva por meio do cálculo das prevalências das variáveis investigadas, utilizou-se a regressão logística binária bruta e ajustada, com os resultados expressos em Odds Ratio (OR) e IC95\%, adotando-se o seguinte modelo hierárquico: no nível distal, encontravam-se as variáveis demográficas e econômicas; no 
intermediário, as variáveis comportamentais e no proximal, a variável de percepção subjetiva de estresse. $\mathrm{Na}$ análise ajustada, foram realizados ajustes para as variáveis do mesmo nível (entrando todas), seguindo para o nível superior somente aquelas que apresentaram um valor de $p \leq 0,20$, mediante a utilização do teste de Wald. Em todas as análises, o valor de significância adotado foi de $p<0,05$.

O estudo foi aprovado pelo Comitê de Ética em Pesquisa da Universidade Federal de Santa Catarina e está de acordo com a resolução 196/96 do Conselho Nacional de Saúde.

\section{Resultados}

Dentre os 5.463 adolescentes que responderam ao questionário, foram excluídos os escolares com idades fora da faixa etária idealizada ( $\mathrm{n}=380)$ e os que não preencheram os questionários completamente $(n=55)$. Desse modo, a amostra final foi composta por 5.028 escolares, com idade média de 17 anos (DP: 1,13; variação: $15-19$ ), sendo 59,3\% do sexo feminino e $34,4 \%$ com nível de renda familiar mensal inferior (até $\mathrm{R} \$ 500,00$, equivalente a 2,5 salários mínimos em $1^{\circ}$ de abril de 2002).

A prevalência de autopercepção negativa de saúde dos adolescentes foi de $14,4 \%$ (17,6\% para as moças e $9,6 \%$ para os rapazes; $p<0,0001)$. As proporções e a OR bruta foram maiores nos adolescentes pertencentes a famílias de menor renda, tabagistas, etilistas, que experimentaram drogas ou abstiveram-se em responder e com autopercepção negativa de estresse (Tabela 1).

$\mathrm{Na}$ análise ajustada, a OR foi maior nas moças (OR: 1,99; IC95\%: 1,66-2,38), nos adolescentes pertencentes a famílias de menor renda (OR: 1,54; IC95\%: 1,08-2,20), nos fumantes (OR: 2,00; IC95\%: 1,35-2,94) e naqueles que referiram perceber negativamente o estresse (OR: 3,36; IC95\%: 2,74-4,12).

Tabela 1 - Prevalência de autopercepção negativa de saúde (ANS) e análises de Odds Ratio (OR) bruta e ajustada em adolescentes catarinenses. Santa Catarina, Brasil, 2001-2002

\begin{tabular}{|c|c|c|c|c|c|c|}
\hline & \multicolumn{4}{|c|}{ Análise bruta } & \multicolumn{2}{|c|}{ Análise ajustada ${ }^{a}$} \\
\hline & $\mathbf{n}$ & $\%$ ANS & OR (IC95\%) & Valor de $p$ & OR (IC95\%) & Valor de $p$ \\
\hline Sexo & & & & $<0,001$ & & $<0,001$ \\
\hline Masculino & 2.000 & 9,6 & 1 & & $1^{\mathrm{b}}$ & \\
\hline Feminino & 2.953 & 17,6 & $2,00(1,68-2,39)$ & & $1,99(1,66-2,38)$ & \\
\hline Faixa etária & & & & 0,938 & & 0,808 \\
\hline 15 e 16 anos & 2.424 & 14,3 & 1 & & $1^{\mathrm{b}}$ & \\
\hline 17 a 19 anos & 2.529 & 14,4 & $1,00(0,85-1,18)$ & & $1,02(0,86-1,20)$ & \\
\hline Renda & & & & $<0,001$ & & 0,001 \\
\hline$\geq R \$ 2.000,00$ & 366 & 10,9 & 1 & & $1^{\mathrm{b}}$ & \\
\hline$R \$ 1.001,00$ a $2.000,00$ & 1.030 & 11,7 & $1,07(0,73-1,57)$ & & $1,02(0,69-1,49)$ & \\
\hline $\mathrm{R} \$ 501,00$ a $1.000,00$ & 1.805 & 13,4 & $1,25(0,88-1,79)$ & & $1,17(0,82-1,68)$ & \\
\hline$\leq \mathrm{R} \$ 500,00$ & 1.661 & 17,4 & $1,71(1,20-2,44)$ & & $1,54(1,08-2,20)$ & \\
\hline Atividade física & & & & 0,527 & & 0,608 \\
\hline Ativos & 2.671 & 14,8 & 1 & & $1^{c}$ & \\
\hline Insuficientemente ativos & 1.070 & 14,0 & $0,93(0,76-1,14)$ & & $0,94(0,75-1,17)$ & \\
\hline Tabagismo & & & & $<0,001$ & & $<0,001$ \\
\hline Não & 4.063 & 13,0 & 1 & & $1^{c}$ & \\
\hline Ex-fumante & 292 & 21,2 & $1,80(1,34-2,41)$ & & $1,82(1,29-2,58)$ & \\
\hline Fumante & 266 & 25,6 & $2,29(1,71-3,06)$ & & $2,00(1,35-2,94)$ & \\
\hline Uso de bebidas alcoólicas & & & & 0,007 & & 0,233 \\
\hline Não & 1.574 & 12,4 & 1 & & $1^{c}$ & \\
\hline Sim & 3.330 & 15,3 & $1,27(1,06-1,52)$ & & $1,14(0,91-1,43)$ & \\
\hline Experiência com drogas & & & & $<0,001$ & & 0,141 \\
\hline Não & 4.265 & 13,4 & 1 & & $1^{c}$ & \\
\hline Não quero responder & 100 & 23,0 & $1,92(1,19-3,09)$ & & $1,70(0,94-3,04)$ & \\
\hline Sim & 495 & 18,8 & $1,49(1,17-1,89)$ & & $1,19(0,85-1,67)$ & \\
\hline Autopercepção de estresse & & & & $<0,001$ & & $<0,001$ \\
\hline Positiva & 4.174 & 11,2 & 1 & & $1^{\mathrm{d}}$ & \\
\hline Negativa & 686 & 34,0 & $4,09(3,40-4,92)$ & & $3,36(2,74-4,12)$ & \\
\hline
\end{tabular}

a Modelo hierárquico: Nível 1 (sexo, faixa etária e renda);Nível 2 (sexo, renda, atividade física, tabagismo, consumo de bebidas alcoólicas e experiência em uso de drogas); Nível 3 (sexo, renda, tabagismo, experiência em uso de drogas e autopercepção de estresse); ${ }^{\text {b } C o n t r o l a n d o ~ p a r a ~}$ variáveis do Nível 1; ' Controlando para variáveis do Nível 2; ' Controlando para variáveis do Nível 3. IC95\%: intervalo de confiança de 95\%. 
$\mathrm{Na}$ estratificação por sexo, observou-se que, nos rapazes, a autopercepção negativa de saúde foi associada àqueles que se abstiveram da resposta para o uso de drogas ilícitas e com autoavaliação negativa de estresse (Tabela 2). Nas moças, a autopercepção negativa de saúde foi associada à condição de ex-fumante e autopercepção negativa de estresse (Tabela 3).

\section{Discussão}

Ao confrontar os resultados deste estudo com os de outras pesquisas conduzidas com adolescentes, observou-se que a prevalência de autopercepção negativa de saúde encontrada no presente trabalho foi inferior à identificada em Istambul, na Turquia $(21,6 \%)^{(15)}$, e superior aos valores encontrados na Noruega $(12,0 \%)^{(8)}$. Essas diferenças podem ocorrer em função dos aspectos socioeconômicos e culturais, haja vista que as condições educacionais, sociais e de saúde estão solidificadas na Noruega e encontram-se em transformação na Turquia.

No Brasil, a Pesquisa Nacional por Amostras de Domicílios (PNAD) ${ }^{(6)}$ demonstrou que a prevalência de autopercepção negativa de saúde, no grupo de 14 a 44 anos, foi de $1,85 \%$, com resultados indicando a importância da educação formal na autoavaliação de saúde. Além disso, um estudo caso-controle conduzido no Distrito Federal revelou que a proporção de autoavaliação negativa de saúde foi de 29,4\% nos adolescentes que relataram lesões (danos físicos ou ferimentos) e de $25,5 \%$ naqueles que não as relataram ${ }^{(16)}$.

Destaca-se que a prevalência de autopercepção negativa de saúde dos adolescentes catarinenses $(14,4 \%)$ foi próxima à proporção encontrada em trabalhadores da indústria $(14,8 \%)$ do mesmo Estado ${ }^{(17)}$. Por outro lado, estudos nacionais da PNAD $^{(6)}$, da Pesquisa Mundial de Saúde ${ }^{(18)}$ e do sistema de

Tabela 2 - Prevalência de autopercepção negativa de saúde (PNS) e análises de Odds Ratio (OR) bruta e ajustada em rapazes. Santa Catarina, Brasil, 2001-2002

\begin{tabular}{|c|c|c|c|c|c|c|}
\hline \multirow{3}{*}{ Faixa etária } & \multirow[b]{2}{*}{$\mathbf{n}$} & \multirow[b]{2}{*}{$\%$ ANS } & \multicolumn{2}{|c|}{ Análise bruta } & \multicolumn{2}{|c|}{ Análise ajustada $^{a}$} \\
\hline & & & OR (IC95\%) & Valor de $p$ & OR (IC95\%) & Valor de $p$ \\
\hline & & & & 0,435 & & 0,424 \\
\hline 15 e 16 anos & 918 & 9,0 & 1 & & $1^{\mathrm{b}}$ & \\
\hline 17 a 19 anos & 1.082 & 10,1 & $1,12(0,83-1,52)$ & & $1,13(0,83-1,54)$ & \\
\hline Renda & & & & 0,084 & & 0,087 \\
\hline$\geq \mathrm{R} \$ 2.000,00$ & 189 & 7,4 & 1 & & $1^{\mathrm{b}}$ & \\
\hline $\mathrm{R} \$ 1.001,00$ a $2.000,00$ & 451 & 6,9 & $0,92(0,47-1,77)$ & & $0,92(0,47-1,77)$ & \\
\hline $\mathrm{R} \$ 501,00$ a $1.000,00$ & 746 & 9,9 & $1,37(0,75-2,49)$ & & $1,37(0,75-2,49)$ & \\
\hline$\leq \mathrm{R} \$ 500,00$ & 580 & 11,2 & $1,57(0,86-2,88)$ & & $1,57(0,86-2,87)$ & \\
\hline Atividade física & & & & 0,528 & & 0,652 \\
\hline Ativos & 1.046 & 9,7 & 1 & & $1^{\mathrm{c}}$ & \\
\hline Insuficientemente ativos & 409 & 10,8 & $1,12(1,77-1,64)$ & & $1,10(0,72-1,68)$ & \\
\hline Tabagismo & & & & $<0,0001$ & & 0,068 \\
\hline Não & 1.613 & 8,4 & 1 & & $1^{\mathrm{c}}$ & \\
\hline Ex-fumante & 111 & 11,7 & $1,45(0,79-2,65)$ & & $1,13(0,54-2,37)$ & \\
\hline Fumante & 112 & 21,4 & $2,98(1,83-4,84)$ & & $2,09(1,12-3,92)$ & \\
\hline Uso de bebidas alcoólicas & & & & 0,066 & & 0,223 \\
\hline Não & 537 & 7,4 & 1 & & $1^{\mathrm{c}}$ & \\
\hline Sim & 1.444 & 10,2 & $1,40(0,97-2,02)$ & & $1,36(0,82-2,23)$ & \\
\hline Experiência com drogas & & & & $<0,0001$ & & 0,006 \\
\hline Não & 1.651 & 8,3 & 1 & & $1^{\mathrm{c}}$ & \\
\hline Não quero responder & 40 & 22,5 & $3,20(1,49-6,87)$ & & $3,82(1,56-9,37)$ & \\
\hline Sim & 265 & 14,7 & $1,90(1,30-2,79)$ & & $1,61(0,94-2,75)$ & \\
\hline Autopercepção de estresse & & & & $<0,0001$ & & $<0,0001$ \\
\hline Positiva & 1.760 & 7,8 & 1 & & $1^{\mathrm{d}}$ & \\
\hline Negativa & 182 & 26,4 & $4,21(2,90-6,11)$ & & $3,51(2,31-5,35)$ & \\
\hline
\end{tabular}

${ }^{a}$ Modelo Hierárquico: Nível 1 (faixa etária e renda); Nível 2 (renda, atividade física, tabagismo, consumo de bebidas alcoólicas e experiência em uso de drogas); Nível 3 (renda, tabagismo, experiência em uso de drogas e autopercepção de estresse); ${ }^{\circ}$ Controlando para variáveis do Nível 1;

${ }^{\mathrm{c}}$ Controlando para variáveis do Nível 2; d Controlando para variáveis do Nível 3. IC95\%: intervalo de confiança de 95\%. 
Tabela 3 - Prevalência de autopercepção negativa de saúde (ANS) e análises de Odds Ratio (OR) bruta e ajustada em moças. Santa Catarina, Brasil, 2001-2002

\begin{tabular}{|c|c|c|c|c|c|c|}
\hline & \multicolumn{4}{|c|}{ Análise bruta } & \multicolumn{2}{|c|}{ Análise ajustada $^{a}$} \\
\hline & $\mathbf{n}$ & $\%$ ANS & OR (IC95\%) & Valor de $p$ & OR (IC95\%) & Valor de $p$ \\
\hline Faixa etária & & & & 0,947 & & 0,838 \\
\hline 15 e 16 anos & 1.506 & 17,5 & 1 & & $1^{b}$ & \\
\hline 17 a 19 anos & 1.447 & 17,6 & $1,00(0,83-1,21)$ & & $0,98(0,80-1,18)$ & \\
\hline Renda & & & & 0,005 & & 0,005 \\
\hline$\geq \mathrm{R} \$ 2.000,00$ & 177 & 14,7 & 1 & & $1^{\mathrm{b}}$ & \\
\hline $\mathrm{R} \$ 1.001,00$ a $2.000,00$ & 579 & 15,4 & $1,05(0,65-1,69)$ & & $1,05(0,65-1,69)$ & \\
\hline $\mathrm{R} \$ 501,00$ a $1.000,00$ & 1.059 & 15,8 & $1,08(0,69-1,70)$ & & $1,08(0,69-1,70)$ & \\
\hline$\leq \mathrm{R} \$ 500,00$ & 1.081 & 20,7 & $1,51(0,97-2,36)$ & & $1,52(0,97-2,36)$ & \\
\hline Atividade física & & & & 0,228 & & 0,377 \\
\hline Ativos & 1.625 & 18,2 & 1 & & $1^{c}$ & \\
\hline Insuficientemente ativos & 661 & 16,0 & $0,86(0,67-1,09)$ & & $0,89(0,68-1,15)$ & \\
\hline Tabagismo & & & & $<0,0001$ & & $<0,0001$ \\
\hline Não & 2.450 & 16,1 & 1 & & $1^{c}$ & \\
\hline Ex-fumante & 181 & 27,1 & $1,93(1,37-2,73)$ & & $2,09(1,40-3,13)$ & \\
\hline Fumante & 154 & 28,6 & $2,08(1,44-3,00)$ & & $1,89(1,15-3,10)$ & \\
\hline Uso de bebidas alcoólicas & & & & 0,004 & & 0,496 \\
\hline Não & 1.037 & 14,9 & 1 & & $1^{\mathrm{c}}$ & \\
\hline Sim & 1.886 & 19,2 & $1,35(1,10-1,66)$ & & $1,09(0,84-1,40)$ & \\
\hline Experiência com drogas & & & & 0,016 & & 0,978 \\
\hline Não & 2.614 & 16,7 & 1 & & $1^{c}$ & \\
\hline Não quero responder & 60 & 23,3 & $1,52(0,82-2,79)$ & & $1,05(0,47-2,31)$ & \\
\hline Sim & 230 & 23,5 & $1,53(1,11-2,11)$ & & $1,04(0,67-1,60)$ & \\
\hline Autopercepção de estresse & & & & $<0,0001$ & & $<0,0001$ \\
\hline Positiva & 2.414 & 13,6 & 1 & & $1^{d}$ & \\
\hline Negativa & 504 & 36,7 & $3,68(2,97-4,57)$ & & $3,36(2,67-4,23)$ & \\
\hline
\end{tabular}

a Modelo hierárquico: Nível 1 (faixa etária e renda); Nível 2 (renda, atividade física, tabagismo, consumo de bebidas alcoólica e experiência em uso de drogas); Nível 3 (renda, tabagismo e autopercepção de estresse); ${ }^{\mathrm{b}}$ Controlando para variáveis do Nível $1 ;{ }^{\circ}$ Controlando para variáveis do Nível 2; d Controlando para variáveis do Nível 3. IC95\%: intervalo de confiança de 95\%.

vigilância nacional ${ }^{(2)}$ demonstraram prevalências bastante inferiores (4,3, 9,2 e 4,5\%, respectivamente) aos resultados observados no presente estudo. Contudo, as análises da autoavaliação de saúde utilizadas pelo sistema de vigilância consideraram a autopercepção negativa como sendo a resposta "ruim", diferentemente dos demais estudos ${ }^{(6,17,18)}$ e desta pesquisa, que consideraram "regular" e "ruim" como avaliação negativa.

No presente estudo, as moças apresentaram maior autopercepção negativa de saúde do que os rapazes. Resultados similares foram observados em outros estudos com adolescentes $^{(8,10,12,15,19-21)}$. Acredita-se que, culturalmente, as moças façam mais exames de rotina e visitem com maior frequência um médico, o que aumenta a chance de diagnóstico precoce de alguma doença. Além disso, elas apresentam maior sensibilidade para detectar alterações fisiológicas ${ }^{(22)}$ e considerar hábitos inadequados para a saúde ${ }^{(23)}$.
Apesar de não ter sido observada associação estatisticamente significante entre a faixa etária e o desfecho investigado neste estudo, outros autores observaram que adolescentes com idade superior tendem a avaliar mais negativamente a saúde, quando comparados aos mais jovens ${ }^{(9,20)}$. Por outro lado, os adolescentes pertencentes a famílias de menor renda apresentaram maior OR para autopercepção negativa de saúde. Sabe-se que a renda representa um componente determinante no maior acesso a outras formas de atividades de lazer, educação, moradia e serviços de saúde. Consequentemente, um poder aquisitivo superior tende a atuar como um mediador do nível de saúde percebida. De forma semelhante, nas análises conduzidas na Europa, América do Norte, Israel ${ }^{(7)}$ e Turquia ${ }^{(15)}$, adolescentes oriundos de estratos de renda inferiores apresentaram chances superiores de referir negativamente a saúde, quando comparados aos de nível de renda superior. 
Quanto aos comportamentos de risco à saúde, os adolescentes que afirmaram fumar atualmente apresentaram maior OR de referir o desfecho em relação aos não-fumantes. $\mathrm{O}$ mesmo foi observado em outros estudos ${ }^{(8,10,12)}$. Quanto ao hábito de consumir bebidas alcoólicas, pesquisas ${ }^{(9,10)}$ com adolescentes demonstraram relação com a autopercepção negativa de saúde, diferentemente do que foi observado na presente investigação.

Em relação ao consumo de drogas ilícitas, associações com a autopercepção de saúde foram marcantes para os rapazes que referiram não querer responder à questão, sendo a OR para referir negativamente a saúde superior neste grupo quando comparados àqueles que referiram jamais ter experimentado drogas. Essa abstenção de informação revela a dificuldade de se discutir tais questões e o receio de afirmar a possível experiência. O uso de drogas, regular ou esporádico, representa um comportamento relacionado com níveis negativos de saúde percebida ${ }^{(10)}$ e tende a declinar ainda mais ao longo dos anos ${ }^{(24)}$.

Um estudo demonstrou que adolescentes tailandeses que participavam de atividade física vigorosa, de força muscular ou esportiva, apresentaram menor prevalência de autopercepção negativa de saúde ${ }^{(25)}$. Nesta pesquisa, a prática de atividade física não se associou à autopercepção negativa de saúde em adolescentes. Destaca-se que, no presente estudo, a medida de atividade física incorporou minutos de prática em diferentes atividades físicas em níveis moderados a vigorosos, diferentemente de outros que consideraram a prática esportiva $^{(8,11)}$ e os exercícios físicos ${ }^{(8)}$.

Além dos aspectos comportamentais que mostraram relevância para a forma de avaliação subjetiva de saúde, o nível de estresse percebido, neste estudo e em pesquisa com adolescentes canadenses ${ }^{(12)}$, apresentou relação significante com a autopercepção de saúde. Sabidamente, essa é uma fase de formação e inserção do indivíduo em situações diferenciadas, como o mercado de trabalho e estudos, além do aumento das responsabilidades pessoais.

\section{Referências bibliográficas}

1. Kasmel A, Helasoja V, Lipand A, Prättälä R, Klumbiene J, Pudule I. Association between health behaviour and self-reported health in Estonia, Finland, Latvia and Lithuania. Eur J Public Health 2004;14:32-6.

2. Brasil. Ministério da Saúde. Vigilância de fatores de risco e proteção para doenças crônicas por inquérito telefônico: estimativas sobre freqüência e distribuição sócio-demográfica de fatores de risco e proteção para doenças crônicas nas capitais dos 26 estados brasileiros e no Distrito Federal em 2006. Brasília: Ministério da Saúde, 2009.
Dentre as limitações da pesquisa, destaca-se que a autopercepção de saúde em adolescentes pode representar o modo como se percebe a saúde física ${ }^{(8,12)}$, embora outros aspectos como a autoestima ${ }^{(8)}$ e o pessimismo e/ou otimismo ${ }^{(26)}$ sejam componentes que interfiram na maneira como se autoavalia o estado de saúde. Soma-se a isso o uso de questionário para obter informações sobre comportamentos indesejáveis à saúde, como o uso de drogas ilícitas, tabagismo e consumo de bebidas alcoólicas, que podem ser subestimados, assim como a possibilidade de superestimação dos comportamentos considerados positivos (prática de atividade física) ${ }^{(27)}$. Além disso, as informações provenientes desta pesquisa são derivadas de um estudo de delineamento transversal, o que inviabiliza possíveis relações de causa e efeito ${ }^{(15)}$.

Conclui-se que as moças, os adolescentes de baixa renda, os fumantes e aqueles com autopercepção negativa de estresse apresentaram OR superiores de autoavaliação negativa de saúde. Quanto à análise por sexo, as moças que afirmaram ser ex-fumantes e perceber negativamente o estresse apresentaram maior OR de saúde negativa percebida. Para os rapazes, a autoavaliação negativa de saúde foi observada naqueles que preferiram não responder se haviam experimentado drogas e naqueles que autoavaliaram negativamente o estresse. Sugere-se a elaboração de estratégias de ações destinadas à promoção de hábitos de vida saudáveis, por meio de programas estruturados no ambiente escolar, priorizando acesso às informações e oportunidades para mudanças de comportamentos relacionados à saúde.

\section{Agradecimentos}

Ao Conselho Nacional de Pesquisa (CNPq) pelo financiamento. À Secretaria de Estado da Educação de Santa Catarina, aos diretores, professores e alunos das escolas selecionadas para coleta de dados e aos membros do Núcleo de Pesquisa em Atividade Física e Saúde da Universidade Federal de Santa Catarina, que participaram da coleta dos dados.

3. Svedberg P, Bardage C, Sandin S, Pedersen NL. A prospective study of health, life-style and psychosocial predictors of self-rated health. Eur $\mathrm{J}$ Epidemiol 2006;21:767-76.

4. Kaplan GA, Camacho T. Perceived health and mortality: a nine-year followup of the human population laboratory cohort. Am J Epidemiol 1983;117: 292-304.

5. Idler EL, Angel RJ. Self-rated health and mortality in the NHANESEpidemiologic Follow-up Study. Am J Public Health 1990;80:446-52. 
6. Dachs JNW, Santos APR. Auto-avaliação do estado de saúde no Brasil: análise dos dados da PNAD/2003. Cienc Saude Coletiva 2006;11:887-94.

7. Richter M, Erhart M, Vereecken CA, Zambon A, Boyce W, Nic Gabhainn S. The role of behavioural factors in explaining socio-economic differences in adolescent health: a multilevel study in 33 countries. Soc Sci Med 2009;69:396-403.

8. Breidablik HJ, Meland E, Lydersen S. Self-rated health in adolescence: a multifactorial composite. Scand J Public Health 2008;36:12-20.

9. Johnson PB, Ritcher L. The relationship between smoking, drinking, and adolescents' self-perceived health and frequency of hospitalization: analyses from the 1997 National Household Survey on Drug Abuse. J Adolesc Health 2002;30:175-83.

10. Wade TJ, Vingilis E. The development of self-rated health during adolescence: an exploration of inter - and intra-cohort effects. Can J Public Health 1999;90:90-4.

11. Pastor Y, Balaguer I, Pons D, García-Merita M. Testing direct and indirect effects of sports participation on perceived health in Spanish adolescents between 15 and 18 years of age. J Adolesc 2003;26:717-30.

12. Vingilis ER, Wade TJ, Seeley JS. Predictors of adolescent self-rated health. Analysis of the National Population Health Survey. Can J Public Health 2002;93:193-7.

13. Instituto Nacional de Estudos e Pesquisas Educacionais Anísio Teixeira - INEP [homepage on the Internet]. Censo Escolar [cited 2000 July 20]. Available from: http://www.inep.gov.br/basica/censo/Escolar/Matricula/

14. Prochaska JJ, Sallis JF, Long B. A physical activity screening measure for use with adolescents in primary care. Arch Pediatr Adolesc Med 2001;155:554-9.

15. Erginoz E, Alikasifoglu M, Ercan O, Uysal O, Ercan G, Albayrak Kaymak D et al. Perceived health status in a Turkish adolescent sample: risk and protective factors. Eur J Pediatr 2004;163:485-94.

16. Pattussi MP, Lalloo R, Bassani DG, Olinto MT. The role of psychosocial, behavioural and emotional factors on self-reported major injuries in Brazilian adolescents: a case-control study. Injury 2008;39:561-9.
17. Barros MV, Nahas MV. Health risk behaviors, health status self-assessment and stress perception among industrial workers. Rev Saude Publica 2001;35: 554-63.

18. Szwarcwald CL, Souza-Júnior PR, Esteves MA, Damacena GN, Viacava F. Socio-demographic determinants of self-rated health in Brazil. Cad Saude Publica 2005;21 (Suppl 1):S54-64.

19. Hidalgo I, Garrido G, Hernandez M. Health status and risk behavior of adolescents in the north of Madrid, Spain. J Adolesc Health 2000;27: 351-60.

20. Torsheim T, Currie C, Boyce W, Kalnins I, Overpeck M, Haugland S. Material deprivation and self-rated health: a multilevel study of adolescents from 22 European and North American countries. Soc Sci Med 2004;59:1-12.

21. Heard HE, Gorman BK, Kapinus CA. Family structure and self-rated health in adolescence and young adulthood. Popul Res Policy Rev 2008; 27:773-97.

22. Shadbolt B. Some correlates of self-rated health for Australian women. Am J Public Health 1997;87:951-6.

23. Meurer LN, Layde PM, Guse CE. Self-rated health status: a new vital sign for primary care? WMJ 2001;100:35-9.

24. Kertesz SG, Pletcher MJ, Safford M, Halanych J, Kirk K, Schumacher J et al. Illicit drug use in young adults and subsequent decline in general health: the Coronary Artery Risk Development in Young Adults (CARDIA) Study. Drug Alcohol Depend 2007;88:224-33.

25. Page RM, Suwanteerangkul J. Self-rated health, psychosocial functioning, and health-related behavior among Thai adolescents. Pediatr Int 2009;51:120-5.

26. Appels A, Bosma H, Grabauskas V, Gostautas A, Sturmans F. Self-rated health and mortality in a Lithuanian and a Dutch population. Soc Sci Med 1996;42:681-9.

27. Farias Júnior JC, Mendes JK, Barbosa DB. Associação entre comportamentos de risco à saúde em adolescentes. Rev Bras Cineantropom Desempenho Hum 2007;9:250-6. 